\title{
O IMPACTO NA QUALIDADE DE VIDA APÓS O REPOSICIONAMENTO CIRÚRGICO DA PRÉ-MAXILA EM PORTADORES DE FISSURA LÁBIO PALATAL BILATERAL-ESTUDO DE 50 CASOS
}

\author{
THE LIFE QUALITY IMPACT AFTER SURGICAL PRE MAXILLA REPOSITIONING IN \\ PATIENTS WITH BILATERAL LIP PALATAL CLEFT - STUDY OF 50 CASES
}

\author{
Kelston Ulbricht Gomes ${ }^{1}$; Abrão Rapoport, ECBC-SP²; Carlos Neutzling Lehn²; \\ Odilon Victor Porto Denardin ${ }^{3} ;$ João Luiz Carlini ${ }^{4}$
}

\begin{abstract}
RESUMO: Objetivo: Descrever o protocolo de tratamento no Centro de Atendimento Integral ao Fissurado Lábio-Palatal em Curitiba (CAIF); (b) avaliar as motivações e expectativas em relação ao tratamento e (c) avaliar a percepção de inclusão e integração social obtida pelo procedimento. Método: Estudo observacional, retrospectivo com todos os 50 participantes recrutados entre os pacientes submetidos ao procedimento de reposicionamento de pré-maxila, no período de janeiro de 2003 a julho de 2005 , no Centro de Atendimento Integral ao Fissurado Lábio-Palatal em Curitiba (CAIF), Paraná. Foi aplicado questionário de avaliação Resultados: Com relação ao protocolo de atendimento, os resultados evidenciaram sucesso em $90 \%$ das cirurgias. Quanto às expectativas cirúrgicas e de integração social, estas mostraram que $76 \%$ procuraram a cirurgia por motivos de insatisfação pessoal e, 86\% relataram maior confiança em si mesmo, após a cirurgia. Conclusão: O protocolo de reposicionamento cirúrgico da prémaxila é uma técnica viável para o tratamento dos pacientes portadores de fissura bilateral do processo alveolar; a maioria dos pacientes submetidos ao tratamento de reposicionamento de pré-maxila percebeu o tratamento como continuidade dos cuidados dispensados, participou por indicação médica, com expectativa de melhora no aspecto pessoal e melhora da autoconfiança e, $96 \%$ deles, manifestaram satisfação com os resultados obtidos, o que facilita a inclusão e integração social dos mesmos (Rev. Col. Bras. Cir. 2008; 35(6): 361-367).
\end{abstract}

Descritores: Cirurgia; Maxila; Fenda Labial; Fissura Palatina.

\section{INTRODUÇÃO}

Os benefícios obtidos através da reconstrução do defeito maxilar com enxerto ósseo autógeno, em pacientes com fissura lábio-palatal, são consagrados na literatura principalmente quando se trata de pacientes com fissura unilateral do processo alveolar ${ }^{1-4}$.

Para o tratamento da pré-maxila em pacientes com fissura bilateral existem diversas modalidades descritas na literatura, tais como a completa remoção da pré-maxila, com a justificativa de promover um melhor fechamento do lábio ${ }^{5}$, e o reposicionamento da pré-maxila com enxerto ósseo autógeno, com resultados satisfatórios ${ }^{6,7}$.

A saúde bucal é um componente indissociável da saúde geral, por isso tem havido grande interesse pelo desenvolvimento e utilização de instrumentos de mensuração de qualidade de vida relacionados à saúde, com o objetivo de avaliar o impacto da doença ou deformidade bucal na vida do indivíduo ${ }^{8,9}$.

Sugere-se que os pacientes com fissura bilateral do processo alveolar, e que apresentam projeção exagerada da pré-maxila, apresentam uma maior tendência em serem vítimas de preconceito e dificuldade de relacionamento no grupo social em que vivem. Por isso os benefícios obtidos através da reconstrução do defeito maxilar, não contribuem apenas para a aparência estética, mas vão muito além, podendo restituir à criança sua identidade nos aspectos biológico, psicológico e sociocultural. Assim, procuramos : descrever o protocolo de tratamento no Centro de Atendimento Integral ao Fissurado Lábio-Palatal em Curitiba (CAIF); avaliar as motivações e expectativas em relação ao tratamento; avaliar a percepção de inclusão e integração social obtida pelo procedimento.

\section{MÉTODO}

O estudo foi previamente aprovado pelo Comitê de Ética em Pesquisa do Hospital Heliópolis, em 14 de agosto de 2007, sob o número 552, e envolveu uma amostra de 50 pacientes ( 33 do sexo masculino, correspondendo a $66 \%$ da amostra e 17 do sexo feminino, correspondendo a $17 \%$ da amostra) e portadores de fissura bilateral trans-forame, operados no período de janeiro de 2003 a julho de 2005.

1. Mestre em Ciências da Saúde do Hospital Heliópolis, Hosphel, São Paulo - SP.

2. Cirurgião do Hospital Heliópolis, Hosphel, São Paulo - SP

3. Bioestatístico Hospital Heliópolis, Hosphel, São Paulo - SP

4. Cirurgião Buco-Maxilo-Facial do Centro de Atendimento Integral Lábio-Palatal de Curitiba, PR.

Recebido em 23/05/2008

Aceito para publicação em 07/08/2008

Conflito de interesses: nenhum

Fonte de financiamento: nenhuma

Trabalho realizado no Curso de Pós-Graduação do Hospital Heliópolis, Hosphel, São Paulo e no Centro de Atendimento Integral Lábio-Palatal de Curitiba, PR. 
Todos os pacientes foram submetidos ao protocolo de atendimento proposto pelo CAIF (Tabela 1).

A pesquisa teve início com a aplicação dos questionários no setor de Cirurgia Bucomaxilofacial nas dependências do Centro de Atendimento Integral ao Fissurado LábioPalatal, Curitiba-PR (Tabela 1). Todos foram orientados sobre a pesquisa, assinando o termo de consentimento, autorizando as manobras propostas, assim como a divulgação dos resultados. Os questionários foram aplicados de forma individual pelo pesquisador em uma sala reservada. Foi dada total privacidade às crianças e aos responsáveis para responder às perguntas do questionário e não havia tempo limite para as respostas. As respostas eram, então, submetidas a uma avaliação, juntamente com a pedagoga da Instituição, para verificar, de fato, se o benefício social foi alcançado.

Quanto ao protocolo de tratamento, este envolvia a avaliação ortodôntica, com a instalação de disjuntores na maxila para corrigir a atresia maxilar e procurar melhorar o posicionamento da pré-maxila. A idade para o tratamento cirúrgico foi determinada em função do posicionamento dos caninos superiores e inferiores, entre 8 e 12 anos de idade.

Após o paciente ser considerado apto para o ato cirúrgico, é feito uma consulta pré-operatória, na qual são solicitados exames de sangue com hemograma completo, coagulograma completo, bioquímica do sangue (dosagens de sódio, potássio, creatinina, uréia, glicose) e, exame de elementos anormais e sedimentos de urina. São requisitados ainda, radiografias de face, panorâmica e telerradiografia de perfil e, radiografias intrabucais oclusal superior e periapical de incisivos superiores. A radiografia panorâmica pré-operatória é necessária, principalmente para determinar a posição dos caninos inferiores e a possibilidade de remoção do enxerto da área do mento (Figura 1). Na mesma consulta pré-operatória são realizadas moldagens com alginato da arcada dentária superior e inferior e, através de uma cirurgia simulada nos modelos de gesso, a pré-maxila é cortada recolocada em uma posição mais satisfatória, a qual permite uma forma de arco satisfatória, diminuindo a projeção da pré-maxila e os espaços correspondentes à fissura. Nesta fase, é confeccionado um guia cirúrgico em acrílico autopolimerizável, para ser usado no trans-operatório e auxiliar no posicionamento da pré-maxila. É solicitado ao ortodontista a colagem de bráquetes ortodônticos nos incisivos e molares superiores para ajudar na fixação do guia cirúrgico usando-se fios de aço número 0.

Os pacientes encaminhados ao centro cirúrgico foram submetidos a um procedimento sob anestesia geral e intubação orotraqueal. Logo após, realiza-se a antissepsia de toda a face do paciente com solução de iodo povidine, seguindo com a aposição de campos estéreis sobre o paciente.

Sob anestesia geral, o tamponamento da orofaringe é feito com gazes estéreis para proteção de vias aéreas superiores. A técnica cirúrgica utilizada preconiza infiltração de lidocaína 2\%, com adrenalina diluída 1:200.000 na região a ser operada, com a finalidade de promover vasoconstrição e analgesia. Com uma lâmina de bisturi número 15 , realiza-se a incisão vertical nas bordas da fissura, estendendo posterioriormente com uma incisão intra-sulcular de 01 a 02 dentes distantes da fissura. Nesta região, realiza-se uma incisão relaxante em direção ao fundo de vestíbulo. Realizou-se o mesmo procedimento no lado oposto, seguido do descolamento do retalho mucoperiostal .

Na borda da fissura da pré-maxila fizemos uma incisão vertical e descolamos somente o suficiente para expormos o osso alveolar remanescente. Na porção palatina foi exposto

Tabela 1 - Questionário para avaliação das expectativas dos fissurados lábio-palatinos.

1 - Por que você procurou o tratamento?

a) porque queria mastigar, falar e comer melhor.

b) porque você ficava preocupado (a) com sua aparência física e queria ficar mais bonito(a).

c) porque o médico ou dentista disse que era necessário.

d) você procurou o tratamento sozinho, porque ficava preocupado (a) e envergonhado(a) por causa dos problemas com sua boca.

2- O que você esperava obter de melhora com a cirurgia?

a) Faria mais amigos, ficaria mais relaxado (a) e conversaria mais em casa e na escola.

b) Ficaria mais bonito (a).

c) Suas notas na escola iriam melhorar.

d) Iria se sentir melhor consigo mesmo (a).

3 - Quais foram as mudanças que você notou depois da cirurgia?

a) Ficou mais bonito (a).

b) Sentiu que ficou mais difícil falar algumas palavras.

c) Sentiu que a vida, em geral, ficou melhor depois da correção dos problemas na sua boca.

d) Não observou.

4 - Você está satisfeito (a) com o tratamento?

a) $\mathrm{Sim}$.

b) Não. 


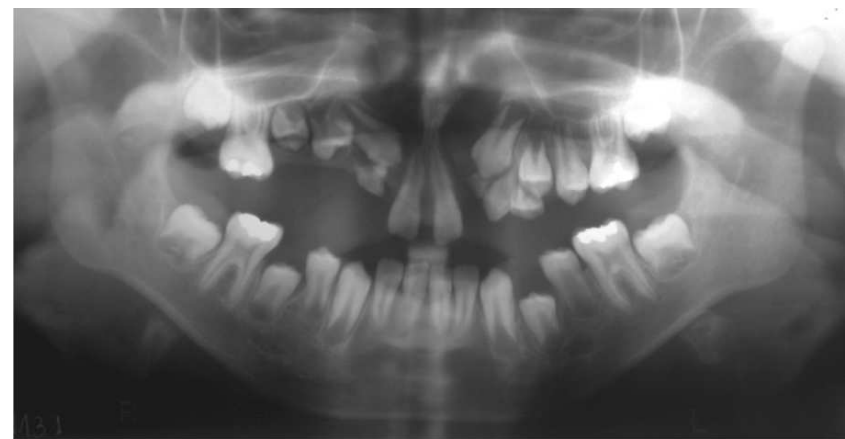

Figura 1 - Radiografia panorâmica pré-operatória evidenciando a posição dos caninos inferiores, a qual permite a remoção de enxerto do mento.

o osso que sustenta a pré-maxila. Com o uso de uma serra reciprocante foi feita a osteotomia na porção posterior à prémaxila. Em seguida foi incisada a mucosa palatina na altura da fissura palatina, mantendo cerca de $1,5 \mathrm{~cm}$ de mucosa palatina aderida na pré-maxila. A pré-maxila foi deslocada anteriormente e, a seguir, dividiu-se a mucosa nasal da bucal, promovendo o fechamento do assoalho nasal bilateral com fio reabsorvível. Uma vez vedado o assoalho nasal, realizamos a sutura da mucosa palatina com o remanescente da mucosa palatina da pré-maxila. O guia cirúrgico foi instalado e a pré-maxila foi reposicionada.

$\mathrm{Na}$ área doadora, quando a sínfise mandibular era utilizada, realiza-se uma incisão em mucosa labial, $1,5 \mathrm{~cm}$ abaixo da linha do vermelhão do lábio inferior, incisando mucosa e músculos, direcionando, em seguida, o bisturi para osso mandibular. Após o descolamento do periósteo, com uma broca de corte rotatória, foi realizado a osteotomia com um desenho apropriado para se adaptar aos defeitos da fissura.

Os blocos de enxertos ósseos foram adaptados às fissuras e fixados com miniplacas retas e parafusos. As miniplacas foram adaptadas para estabilizar os enxertos e a pré-maxila ao remanescente da maxila. O guia cirúrgico, além

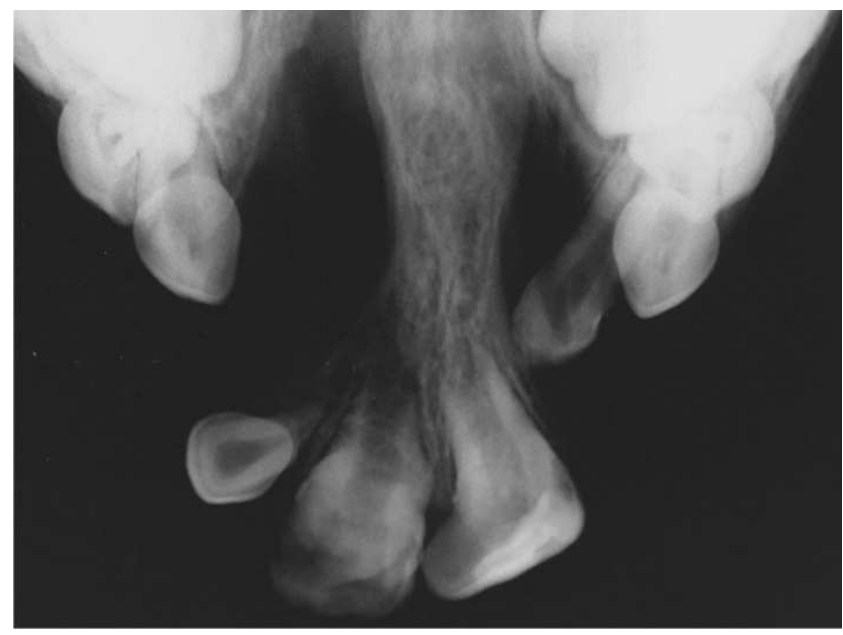

Figura 2 - Radiografia oclusal pré-operatória,evidenciando a presença de fissura bilateral. de fornecer a nova posição da pré-maxila, ajuda também na sustenção e fixação da mesma, e deve permanecer por no mínimo dois meses fixado a nova arcada superior.

O recobrimento do enxerto foi realizado com o retalho mucoperiostal, deslocado através de incisões relaxantes no periósteo da base do retalho. O fechamento da fissura foi promovido com o retalho livre de tensão e a sutura realizada com fio de nylon.

O acompanhamento pós-operatório foi ambulatorial e a sutura foi removida em duas semanas. A remoção do guia cirúrgico foi realizada após dois meses de cirurgia. A remoção das placas e parafusos foi realizada após três meses de cirurgia. Após esse período, o paciente era encaminhado, novamente, para retomar o tratamento ortodôntico.

\section{RESULTADOS}

Dentre os 50 pacientes operados, 45 (90\%) casos tiveram cirurgias com sucesso, os enxertos ósseos integraram e houve o fechamento das fístulas buconasais. Nos cinco pacientes remanescentes $(10 \%)$ o tratamento falhou ou teve sucesso parcial (um paciente com perda bilateral do enxerto; dois pacientes com perdas unilaterais do enxerto e dois casos de necrose da pré-maxila) após a primeira intervenção. Nos três casos de perda de enxerto, os pacientes se submeteram à nova intervenção com sucesso do procedimento. Nos dois casos de perda de pré-maxila por necrose, ocorreram novas cirurgias com enxerto para recuperar a pré-maxila com resultados satisfatórios.

Os parâmetros que permitem caracterizar o sucesso do tratamento foram baseados nas radiografias periapicais e oclusais, realizadas após 06 meses do ato operatório. Foi observado o preenchimento ósseo das fissuras e, ausência de mobilidade da pré-maxila ao exame clínico. Novas radiografias periapicais e oclusais foram realizadas com 12 meses de pósoperatório para se observar a irrupção dos caninos superiores e a movimentação ortodôntica dos dentes adjacentes à fissura (Figuras 2 e 3).

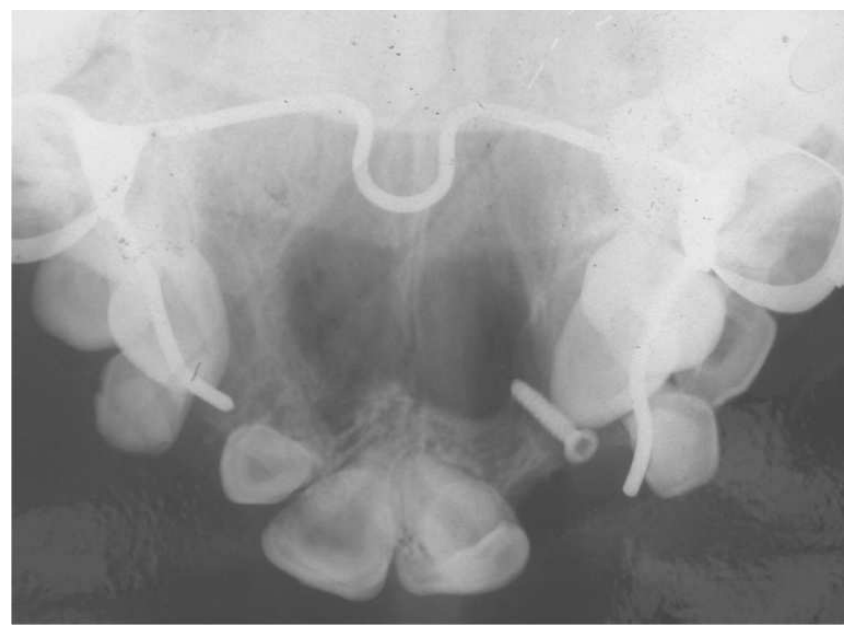

Figura 3 - radiografia oclusal pós-operatória, evidenciando a presença de ponte óssea, na região da fissura previamente enxertada. 
Quanto ao motivo da procura pelo tratamento cirúrgico, $84 \%$ dos pacientes tiveram indicação profissional e apenas $16 \%$ procuraram a cirurgia para melhora de condições estéticas e funcionais. (Tabela 2).

Quanto aos resultados em relação à expectativa de melhora após o tratamento, evidenciou-se que $76 \%$ procuraram pelo tratamento cirúrgico por insatisfação pessoal (estética). Em $18 \%$ dos entrevistados houve esperança por melhora nos relacionamentos sociais e afetivos, e em $6 \%$ houve interesse pela melhora das condições profissionais. (Tabela 3 ).

As mudanças percebidas foram significativas, $86 \%$ relataram maior confiança em si, e $14 \%$ observaram mudanças na aparência física. (Tabela 4 e Figuras 4 e 5).

Quanto à satisfação com os resultados, 96\% afirmaram estar satisfeitos. (Tabela 5).

\section{DISCUSSÃO}

A intervenção cirúrgica em pacientes portadores de fissuras bilaterais causa grande repercussão, principalmente

Tabela 2 - Distribuição de freqüências da variável motivo de procura pelo tratamento, na amostra total.

\begin{tabular}{lcc}
\hline Motivo de Procura & \multicolumn{2}{c}{ Freqüências } \\
\cline { 2 - 3 } & Absoluta(n) & Relativa (\%) \\
\hline Melhora funcional & 3 & $6 \%$ \\
Melhora estética & 5 & $10 \%$ \\
Indicação profissional & 42 & $84 \%$ \\
Iniciativa própria & 0 & $0 \%$ \\
Total & 50 & $100 \%$ \\
\hline
\end{tabular}

Tabela 3 - Distribuição de freqüências da variável expectativa de melhora com o tratamento, na amostra total.

\begin{tabular}{lcc}
\hline Motivo de Procura & \multicolumn{2}{c}{ Freqüências } \\
\cline { 2 - 3 } & Absoluta(n) & Relativa(\%) \\
\hline Social/afetiva & 9 & $18 \%$ \\
Física/ estética & 0 & $0 \%$ \\
Profissional/escolar & 3 & $6 \%$ \\
Insatisfação pessoal & 38 & $76 \%$ \\
Total & 50 & $100 \%$ \\
\hline
\end{tabular}

Tabela 4 - Distribuição de freqüências da variável mudanças percebidas após o tratamento, na amostra total.

\begin{tabular}{lcc}
\hline Motivo de Procura & \multicolumn{2}{c}{ Freqüências } \\
\cline { 2 - 3 } & Absoluta(n) & Relativa (\%) \\
\hline Aparência física & 7 & $14 \%$ \\
Dificuldade na voz & 0 & $0 \%$ \\
Mais confiante & 43 & $86 \%$ \\
Não observado & 0 & $0 \%$ \\
Total & 50 & $100 \%$ \\
\hline
\end{tabular}

quando se trata da pré-maxila, considerando a interferência no crescimento maxilar e os riscos do procedimento. Entretanto, a idade recomendada para realização do enxerto ósseo secundário é entre 8 e $12 \operatorname{anos}^{10}$, sendo que os danos ao crescimento nessa região podem ter sido causados já durante o fechamento primário do lábio e/ou palato ${ }^{11}$. Este conceito também é apoiado por outros autores, que sugerem que o reposicionamento da pré-maxila pode ser realizado antes do término do crescimento facial, sem trazer prejuízos. Eles ainda sugerem que um posterior desenvolvimento de uma relação de classe III, pode ser atribuído a um prognatismo mandibular, e não a uma hipoplasia maxilar $^{12}$. Outro grupo de pesquisadores também parece concordar que a osteotomia da pré-maxila proporciona um bom alinhamento do arco superior e, devolvem uma inclinação mais satisfatória aos incisivos superiores. Sendo assim, sugerem que mais estudos sejam realizados para observação das conseqüências, no longo prazo, sobre o crescimento facial ${ }^{13}$, o que não ocorreu em nosso trabalho.
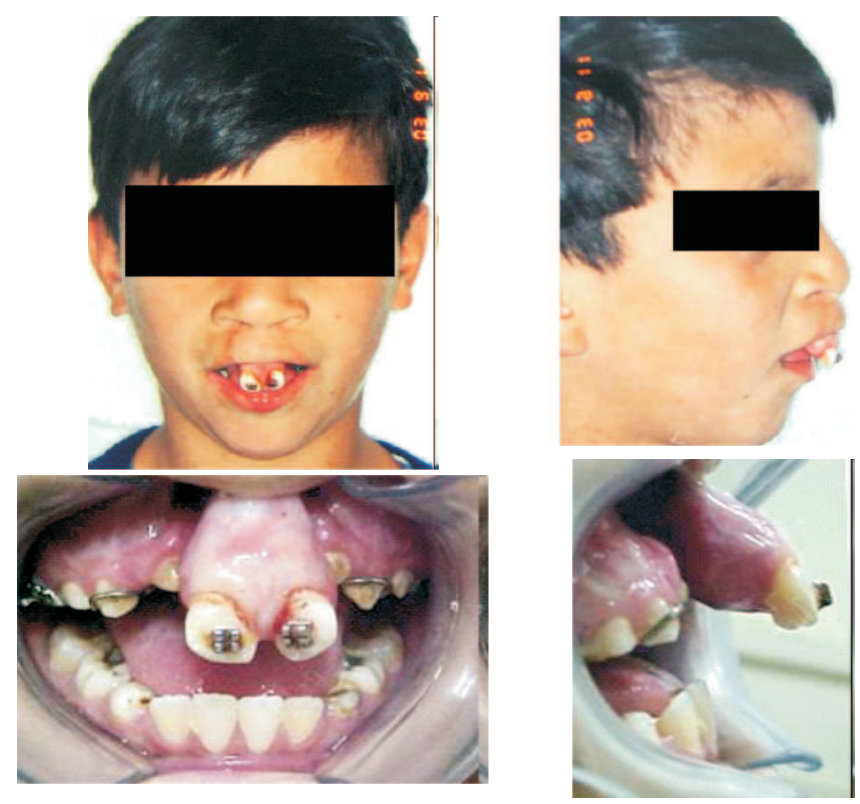

Figura 4 - Aspecto pré-operatório de fissura lábio palatal.
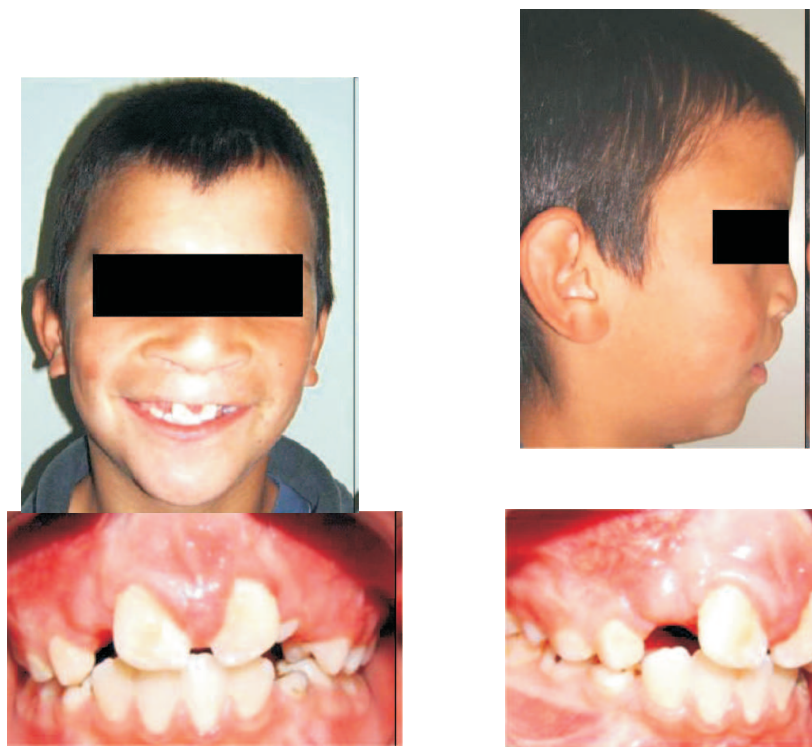

Figura 5 - Aspecto pós-operatório de fissura lábio palatal. 
Tabela 5 - Distribuição de freqüências da variável satisfação com os resultados do tratamento, amostra total.

\begin{tabular}{lcc}
\hline Gênero & \multicolumn{2}{c}{ Freqüiências } \\
\cline { 2 - 3 } & Absoluta (n) & Relativa (\%) \\
\hline Satisfeito & 48 & $96 \%$ \\
Não satisfeito & 2 & $4 \%$ \\
Total & 50 & $100 \%$ \\
\hline
\end{tabular}

Outro aspecto de grande relevância com relação à osteotomia da pré-maxila, desde a sua introdução, diz respeito ao risco de suprimento sanguíneo inadequado para a prémaxila e sua conseqüente perda por necrose ${ }^{14-16}$. Este aspecto do procedimento também está relacionado à utilização correta do método cirúrgico por equipe devidamente treinada e habilitada para sua execução. Falhas técnicas e ou desconhecimento da anatomia regional, podem levar à perda do suprimento sanguíneo do segmento ósseo deslocado e sua conseqüente perda. Quanto ao tipo de enxerto utilizado, várias áreas doadoras podem ser utilizadas, sendo a mandíbula a área que oferece maior quantidade de vantagens, como por exemplo a origem embrionária semelhante ${ }^{17}$, apesar da crista ilíaca ser usada mais recentemente para a enxertia em virtude da maior densidade óssea. O posicionamento dos caninos inferiores foi fundamental, pois dependendo do estágio de erupção dentária, podem dificultar a remoção do enxerto da região de sínfise mandibular (Figura 1), ou seja, é necessário que o estágio de erupção esteja avançado para que haja espaço para realização da osteotomia sem causar danos às raízes dentárias com a broca de corte. Assim em 24 pacientes, o enxerto foi removido da região da sínfise mandibular, e o uso de enxerto de crista ilíaca, em 26 pacientes, em fissuras muito grandes.

Por outro lado, alguns estudos vêm analisando os aspectos psicossociais e os benefícios alcançados com o tratamento cirúrgico em pacientes fissurados bilaterais. Alguns autores acreditam e estabelecem uma grande importância ao complexo maxilofacial na concretização da fisionomia dos seres humanos, principalmente depois de analisarem os portadores de má formação congênita lábio-palatal ${ }^{4,18}$.

Ainda, alguns autores acreditam que as crianças com fissuras labiopalatinas em idade escolar enfrentam com frequiência uma experiência social negativa da reação das pessoas e de outras crianças com relação à aparência física diferente. As dificuldades iniciais tendem a se dissipar com a passagem do tempo e a serem minoradas pelo processo reabilitador, que deve se iniciar tão logo que possível ${ }^{9}$. Esse fato justifica, novamente, a possível influência positiva do procedimento cirúrgico proposto, sobre o bem estar social do paciente em tratamento.

Um outro aspecto a ser destacado, seria a repercussão do tratamento proposto na elevação da auto-estima dos pacientes. A satisfação da necessidade de auto-estima leva o indivíduo a sentir-se confiante, mais útil e necessário ao mundo. A não satisfação produz no indivíduo um sentimento de inferioridade, fraqueza e impotência. A persistência desses sentimentos desencadeará fracassos na sua trajetória ou processos patológicos variados ${ }^{19}$. Alguém cronicamente acometido de baixa auto-estima pode ser uma pessoa que tenha sua saúde comprometida. Problemas relacionados à região bucomaxilofacial acarretam péssimas funções mastigatórias, insatisfação para com suas condições orais e precária qualidade de vida ${ }^{20,21}$.

Desta forma, no presente estudo, tentou-se relacionar as melhoras alcançadas no convívio e desempenho escolar, com a elevação do nível de auto-estima das crianças. Esta correlação teria como embasamento o fato de que é na escola o lugar onde a criança irá enfrentar novos relacionamentos e julgamentos e, sua aparência física será um fator importante nesses julgamentos ${ }^{9}$. Complementando ainda essa idéia, existem autores que defendem a existência de uma identidade pessoal que os outros atribuem entre si por sua aparência física. A pessoa que o individuo se torna é uma construção histórica que inclui a auto-representação e a representação que os outros fazem deles. No corpo se inscreve toda uma série de medos e fantasias que determinam o grau de satisfação do indivíduo consigo mesmo ${ }^{22,23}$. O conceito de auto-estima tem sido estudado e considerado como um importante indicador da saúde mental ${ }^{24-26}$. A crítica, em geral, enfoca a necessidade de aplicação de instrumentos precisos que permitam avaliar a necessidade de auto-estima de cada indivíduo, principalmente crianças. Diante disso, é procedente a realização desse estudo que se caracterizou na avaliação dos níveis de auto-estima em pacientes crianças com fissura bilateral do processo alveolar.

Por outro lado, analisando os instrumentos de mensuração de qualidade de vida em crianças, alguns autores concordam que existe uma falta de consenso a respeito da administração do instrumento de avaliação. Sempre que a criança for hábil em prover dados confiáveis e válidos, a aplicação direta nestes pacientes é a estratégia ideal porque é consistente com a definição de qualidade de vida que enfatiza a perspectiva subjetiva do paciente ${ }^{27}$.

Um instrumento administrado por entrevista pode ser mais dispendioso que o questionário auto-administrado. Para crianças muito jovens ou gravemente debilitadas, os responsáveis podem prover informações que não poderiam ser obtidas de outra forma. Além disso, a perspectiva dos os responsáveis é importante por causa da natureza dependente da relação responsável-criança. É este responsável quem avalia o impacto da saúde da criança e decide se esta deve receber o tratamento, podendo também informar o impacto da doença da criança e do tratamento na dinâmica familiar, o que é parte integral da qualidade de vida da crianç ${ }^{28}$.

Existem diversas desvantagens em usar os pais como respondedores. Primeiro, um relato por procuração é de alguma forma inconsistente como conceito de qualidade de vida, que é definido de acordo com a opinião subjetiva do pacien$\mathrm{te}^{28}$. Segundo, o relato de pais e mães pode não ser equivalente entre $\mathrm{si}^{29,30}$, sendo recomendado que seja feito avaliação dos pais e depois cruzar as informações, com o objetivo de captar possíveis erros ${ }^{28}$. Terceiro, o relato dos responsáveis sobre o impacto da doença nas suas crianças é baseado no conhecimento acerca de como eles mesmos são afetados. Fi- 
nalmente, não é totalmente claro se os pais seriam os adultos mais adequados a responder o questionário ${ }^{29}$, uma vez que algumas crianças podem passar mais tempo com professores, cuidadores ou outro membro da família do que com os pais, e assim outro adulto teria um conhecimento maior acerca do funcionamento social e psicológico da criança ${ }^{28}$.

Devido a esta falta de consenso, alguns pesquisadores têm sugerido obter em conjunto informações de responsáveis e crianças ${ }^{30}$. Esta abordagem pode prover uma informação mais completa de como a doença ou o tratamento impactam na vida das crianças e suas famílias ${ }^{28}$. No entanto, autores ${ }^{31}$ afirmaram que é evidente que ainda se está muito aquém de uma concepção uniforme e universal de qualidade de vida na infância, como também de meios de avaliação deste conceito adaptados ao universo infantil. É prioridade que se tenha clara a necessidade de instituir definições que traduzam os interesses das crianças e do adolescente, e não dos adultos que os avaliam, e que se instaurem métodos de avaliação que captem a percepção do indivíduo a ser avaliado, e não as expectativas e percepções do cuidador, seja ele pai ou profissional de saúde.

Sendo assim, a auto-avaliação da saúde bucal e da satisfação global com a vida proporciona à criança e seus responsáveis a oportunidade de expressar sua concepção pessoal da própria realidade e da sua saúde. Os resultados encontrados nesta pesquisa são referentes às pessoas estudadas, não podendo ser generalizados para toda a população de fissurados bilaterais do Centro de Atendimento Integral ao Fissurado Lábio-Palatal, sendo o tamanho desta amostra o fator limitante deste estudo. A partir da análise dos dados observados nas tabelas, pode-se observar que embora houvesse preocupação com uma melhora estética, a aceitação da cirurgia pela maior parte dos pacientes $(84 \%)$ ocorreu pela sugestão da própria instituição, porque, como já descrito anteriormente, o paciente que faz parte de um tratamento reabilitador longo ambienta-se rapidamente aos procedimentos médicos e técnicos do hospital, o que pode não ser positivo, pois o paciente pode não mais responder ao tratamento com a devida autonomia ${ }^{32}$.

A valorização da aparência física contribuiu para a procura pela cirurgia em $10 \%$ dos pacientes estudados, justificado pelo fato de que em países ocidentais, o belo é valoriza- $\mathrm{do}^{33}$. Apesar de serem os problemas funcionais uma das principais argumentações para a intervenção cirúrgica, os pacientes observam mudanças significativas nas suas relações sociais, pois com a nova apresentação do rosto e da fala, sentemse mais seguros na busca da aceitação pelo grupo em que se inserem $^{24}$.

A maioria dos benefícios, esperados pelos pacientes com o tratamento cirúrgico, indicou a dimensão social, afetiva e busca por satisfação pessoal. Esses fatores, segundo a literatura, são os principais motivos que levam a procura pela cirurgia e que ajudam, encorajando, no processo de adaptação pós-operatória ${ }^{22}$.

Quanto às mudanças percebidas pelos pacientes, os resultados foram condizentes com as expectativas de melhora. Essa fase do tratamento cirúrgico é vista pelos pacientes como uma das últimas cirurgias de uma série de várias. Em decorrência disso, o tratamento cirúrgico provoca no paciente um sentimento de libertação e de estar pronto para o convívio social e para os relacionamentos interpessoais. A intervenção cirúrgica constitui um consentimento concreto para a exteriorização de desejos e vontades reprimidas ${ }^{25}$.

De modo geral, o grupo de pacientes estudado ficou bastante satisfeito com os resultados. De acordo com os relatos, os resultados levaram à harmonia estética, além de modificações nas relações interpessoais e aumento da auto-estima; maior integração social e maior cooperação diante das terapêuticas paralelas a recuperação ${ }^{20}$. Tal afirmativa confirma a eficácia do processo cirúrgico e leva a constatar que os pacientes foram beneficiados com o tratamento.

Em síntese, observamos que: o protocolo de reposicionamento cirúrgico da pré-maxila é uma técnica viável para o tratamento dos pacientes portadores de fissura bilateral do processo alveolar. A maioria dos pacientes submetidos ao tratamento de reposicionamento de maxila percebeu o tratamento como continuidade dos cuidados dispensados, participou por indicação médica, com expectativa de melhora no aspecto pessoal e melhora da autoconfiança. $96 \%$ dos pacientes submetidos ao tratamento cirúrgico proposto manifestaram satisfação com os resultados obtidos, o que facilita a inclusão e integração social dos mesmos.

\footnotetext{
ABSTRACT

Background: To describe the assistance protocol, at the Center for Integral Assistance of Cleft Lip and Palate Patients (CAIF); to assess the expectations and motivations in respect to treatment; to evaluate the social integration and inclusion obtained through the procedure. Methods: a retrospective study with all the 50 patients recruited among those submitted to the procedure of premaxilla repositioning, at the period from January 2003 to july 2005, at the Center for Integral Assistance of Cleft Lip and Palate Patients (CAIF), Paraná. A questionnaire was applied. Results: regarding the assistance protocol, the results showed success in $90 \%$ of surgeries. Concerning surgical and social integration expectations, it was showed that $76 \%$ looked after surgery for personal satisfaction reasons and, $86 \%$ related better self trust after surgery. Conclusion: the surgical protocol of premaxilla repositioning is a viable technique for the treatment of patients with bilateral cleft of the alveolar process; the majority of patients submitted to premaxilla repositioning perceived the treatment as continuity of the care received, participated due to medical indication, expecting improvement at personal aspect and in self trust and, $96 \%$ of them, related satisfaction with results, which facilitates integration and social inclusion.
}

Key words: Surgery; Maxilla; Cleft Lip; Cleft Palate. 


\section{REFERÊNCIAS}

1. Boyne PJ. Autogenous cancellous bone and marrow transplants. Clin. Orthop Relat Res. 1970; 73:199-209.

2. Boyne PJ, Sands NR. Secondary bone grafting of residual alveolar and palatal clefts. J Oral Surg. 1972; 30(2):87-92.

3. Spina V. A proposed modification for the classification of cleft lip and palate. Cleft Palate J. 1973; 10:251-2.

4. Andrade D, Angerami ELS. A auto-estima em adolescentes com e sem fissuras de lábio e/ou de palato. Rev Latino-Am Enfermagem.. 2001; 9(6):37-41.

5. Aburezq H, Daskalogiannakis J, Forrest C. Management of the prominent premaxilla in bilateral cleft lip and palate. Cleft Palate Craniofac J. 2006; 43(1): 92-5.

6. Bergland O, Semb G, Abyholm F. Elimination of the Residual Alveolar Cleft by Secondary Bone Grafting and Subsequent Orthodontic Treatment. Cleft Palate J. 1986; 23(3):175-205.

7. Feller C, Antoniazzi JH, Galiotti Filho J, Durão AS, Brunetti RF, Benvenga MN, Tumenas I, Sgrinhelli MR, Cielho H. Odontologia: essencial para a qualidade de vida. Rev Assoc Paul Cirur Dent. 1997; 51(6):514- 521.

8. Bortoli D, Locatelli FA, Fadel CB, Baldani MH. Associação entre Percepção de Saúde Bucal e Indicadores Clínicos e Subjetivos: Estudo em Adultos de um Grupo de Educação Continuada da Terceira Idade. Publ. UEPG Ci. Biol. Saúde, 9 : 55-65, 2003.

9. Amaral VLAR. Vivendo com uma face atípica: influência da deformidade facial e auto e hetero conceitos e na realização acadêmica de crianças de 6 a 12 anos [dissertação]. São Paulo (SP): Universidade de São Paulo; 1986.

10. Sindet-Pedersen S, Enemark H. Comparative study of secondary and late secondary bone-grafting patients with residual cleft defects. Short-term evaluation. Int J Oral Surg. 1985; 14(5):38498.

11. Freihofer HPM, Kuijpers-Jagtman AK. Early secondary osteoplastic closure of the residual alveolar cleft in combination with orthodontic treatment. J Craniomaxillofac Surg. 1989; 17 Suppl 1:26-7.

12. Padwa BL, Sonis A, Bagheri S, Mulliken JB. Children with repaired bilateral cleft lip/palate: effect of age at premaxillary osteotomy on facial growth. Plast Reconstr Surg. 1999; 104(5):1261-9.

13. Heidbuchel K, Kuijpers-Jagtman AM, Freihofer H. An orthodontic and cephalometric study on the results of the combined surgical-orthodontic approach of the protruded premaxilla in bilateral clefts. J Craniomaxillofac Surg. 1993; 21(2):60-6.

14. Brouns J, Egyedi P. Osteotomy of the premaxilla. J Maxillofac Surg. 1980; 8(3):182-6.

15. Banks P. The surgical anatomy of secondary cleft lip and palate deformity and its significance in reconstruction. Br J Oral Surg. 1983; 21(2):78-93.

16. Scott JK, Webb RM, Flood TR. Premaxillary osteotomy and guided tissue regeneration in secondary bone grafting in children with bilateral cleft lip and palate. Cleft Palate Craniofac J. 2007; 44(5):469-75.

17. Koole R, Bosker H, Dussen NVD. Late secondary autogenous bone grafting in cleft patients comparing mandibular (ectomesenchymal) and iliac crest (mesenchymal) grafts. J Craniomaxillofac Surg. 1989; 17 Suppl 1:28-30.

18. Leonard BJ, Brust JD, Abrahams G, Sielaff B. Self-concept of children and adolescents with cleft lip and/or palate. Cleft Palate Craniofac J. 1991; 28(4):347-53.
19. Maslow AH. Motivation and personality. $2^{\text {nd }}$ ed. New York: Harper \& How; 1970.

20. Campos RG. Eu tenho a força. Rev Viver. 2001; 10:17-26.

21. Carvalho LEP. Avaliação do nível de satisfação, capacidade, eficiência e performances mastigatórias em pacientes reabilitados com próteses fixas totais inferiores sobre implantes, sob carga imediata [dissertação]. Bauru (SP): Universidade do Sagrado Coração; 2002.

22. Alves MCR. Aspectos psicológicos das intervenções cirúrgicas na área odontológica. Odontol Mod. 1985;12(2):48-52.

23. Romero E. O corpo vivido. In: ___ As dimensões da vida humana: existência e experiência. São José dos Campos: Novos Horizontes; 1998. p.133-81.

24. Fadiman J, Frager R. Teoria da personalidade. São Paulo: Harbra; 1979.

25. Wolf SMR. O significado psicológico da perda dos dentes em sujeitos adultos. Rev Assoc Paul Cir Dent. 1998; 52(4): 307-16.

26. Cano MAT, Ferriani MGC, Alves AC, Nakata CY. A produção do conhecimento sobre adolescência na enfermagem: período de 1983 a 1996. Rev Latinoam Enfermagem. 1998; 6(1):91-7.

27. Eiser C, Mohay H, Morse R. The measurement of quality of life in young children. Child Care Health Dev. 2000; 26(5):401-14.

28. Matza LS, Swensen AR, Flood EM, Secnik K, Leidy NK. Assessment of health-related quality of life in children: a review of conceptual, methodological, and regulatory issues. Value Health. 2004; 7(1):79-92.

29. Landgraf JM, Abetz LN. Measuring health outcomes in pediatric populations: issue in psychometrics and application. In: Spilker B, editors. Quality of life and pharmacoeconomics in clinical trials. Philadelphia: Lippincott-Raven; 1996.

30. Eiser C, Morse R. Can parents rate their child's health-related quality of life? Results of a systematic review. Qual Life Res. 2001; 10(4):347-57.

31. Assumpção Jr. FB, Kuczynski E, Sprovieri MH, Aranha EMG. Escala de avaliação de qualidade de vida (AUQEI Autoquestionnaire Qualité de Vie Enfant Imagé) validade e confiabilidade de uma escala para qualidade de vida em crianças de 4 a 12 anos. Arq Neuro-Psiquiatr. 2000; 58(1):119-27.

32. Veronez, FS, Tavano LDA. Modificações psicossociais observadas pós-cirurgia ortognática em pacientes com e sem fissuras labiopalatinas. Arq Ciênc Saúde. 2005;12(3):133-7.

33. Garvill J, Garvill H, Kahnberg KE, Lundgren S. Psychological factors in orthognathic surgery. J Craniomaxillofac Surg. 1992; 20(1):28-33.

Como citar este artigo:

Gomes KU, Rapoport A, Lehn CN, Denardin OV, Carlini JL. O impacto na qualidade de vida do reposicionamento cirúrgico da prémaxila em portadores de fissura lábio palatal bilateral - estudo de 50 casos. Rev Col Bras Cir. [periódico na Internet] 2008; 35(6). Disponível em URL: http://www.scielo.br/rcbc

Endereço para correspondência:

Abrão Rapoport

Rua Iramaia, 136 - Jardim Europa

01450-020 - São Paulo - SP

E-mail: arapoport@terra.com.br 